\title{
XSP: Across-Stack Profiling and Analysis of Machine Learning Models on GPUs
}

\author{
Cheng $\mathrm{Li}^{1 *}$, Abdul Dakkak ${ }^{1 *}$, Jinjun Xiong ${ }^{2}$, Wei Wei ${ }^{3}$, Lingjie $\mathrm{Xu}^{4}$, Wen-mei $\mathrm{Hwu}^{1}$ \\ ${ }^{1}$ University of Illinois Urbana-Champaign, ${ }^{2}$ IBM T. J. Watson Research Center, ${ }^{3}$ Alibaba, ${ }^{4}$ Biren Research (formerly at Alibaba) \\ \{cli99, dakkak,w-hwu\}@illinois.edu, jinjun@us.ibm.com,w.wei@alibaba-inc.com, lingjie@birentech.com
}

\begin{abstract}
There has been a rapid proliferation of machine learning/deep learning (ML) models and wide adoption of them in many application domains. This has made profiling and characterization of ML model performance an increasingly pressing task for both hardware designers and system providers, as they would like to offer the best possible system to serve ML models with the target latency, throughput, cost, and energy requirements while maximizing resource utilization. Such an endeavor is challenging as the characteristics of an ML model depend on the interplay between the model, framework, system libraries, and the hardware (or the HW/SW stack). Existing profiling tools are disjoint, however, and only focus on profiling within a particular level of the stack, which limits the thoroughness and usefulness of the profiling results.

This paper proposes XSP - an across-stack profiling design that gives a holistic and hierarchical view of ML model execution. XSP leverages distributed tracing to aggregate and correlate profile data from different sources. XSP introduces a leveled and iterative measurement approach that accurately captures the latencies at all levels of the HW/SW stack in spite of the profiling overhead. We couple the profiling design with an automated analysis pipeline to systematically analyze 65 state-of-the-art ML models. We demonstrate that XSP provides insights which would be difficult to discern otherwise.
\end{abstract}

\section{INTRODUCTION}

Machine learning/deep learning (ML) models are increasingly being used to solve problems across many domains such as image classification, object detection, machine translation, etc. This has resulted in a surge of interest in optimizing and deploying these models on many hardware types including commodity servers, accelerators, reconfigurable hardware, mobile/edge devices, and ASICs. As a result, there is an increasing need to profile and understand the performance of ML models.

Characterizing ML model inference is complex as its performance depends on the interplay between different levels of the HW/SW stack - frameworks, system libraries, and hardware platforms. Figure 1 shows an example model inference pipeline on GPUs. At the top, there is the $\mathbf{0}$ modellevel evaluation pipeline. Components at the model-level include input pre-processing, model prediction, and output postprocessing. Within the model prediction step are the (2) layerlevel components - layer operators including convolution (Conv), batch normalization (BN), softmax, etc. Within each layer are the $3 \mathrm{GPU}$ kernel-level components - a sequence

\footnotetext{
*The two authors contributed equally to this paper.
}

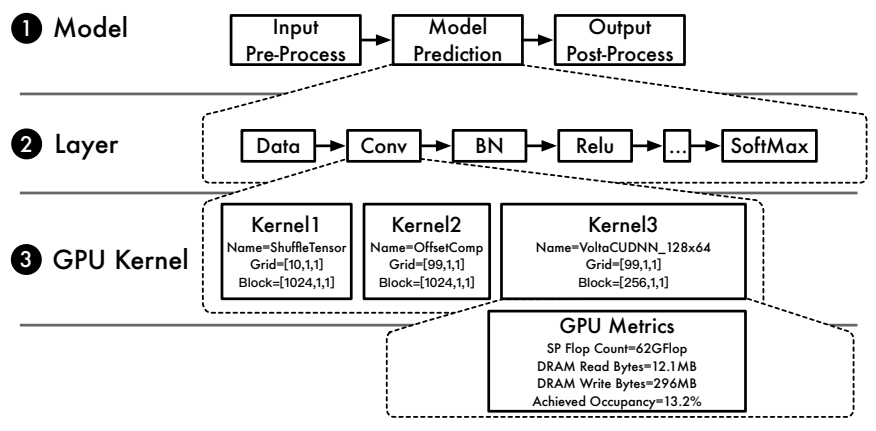

Fig. 1. The model-, layer-, and GPU kernel-level profile of MLPerf_ResNet50_v1.5 (Table VIII) on Tesla_V100 (Table VII) with batch size 256 using NVIDIA GPU Cloud TensorFlow v19.06. The layers executed are data (Data), convolution (Conv), batch normalization (BN), relu (Relu), etc. The 3 GPU kernels from the first Conv layer are shown along with the GPU metrics of Kernel 3.

of CUDA API calls or GPU kernels invoked by the layer. Because of the complexities of model inference, one needs a holistic view of the execution to identify and locate performance bottlenecks.

Existing profiling tools or methods only provide a partial view of model execution. To capture a holistic view of model execution, one has to switch between an array of tools. Take the current ML profiling on GPUs for example. To measure the model-level latency, one inserts timing code around the model prediction step of the inference pipeline. To capture the layer-level information, one uses the ML framework's profiling capabilities [1], [2]. And, to capture GPU kernel information, one uses GPU profilers such as NVIDIA's nvprof [3] or Nsight [4]. The output profiles from the different tools are disjoint; e.g., the GPU kernels are not correlated with the layers. As a result, one cannot construct Figure 1 and identify that the three GPU kernels shown come from the first Conv layer, for example. This same issue exists when profiling ML model execution on CPUs.

To correlate profiled events with model layers, vendors modify ML frameworks and instrument them to work with their profilers. For example, NVIDIA GPU Cloud [5] (NGC) hosts frameworks which are instrumented with NVTX [6] markers. The NVTX markers are added around each layer in the framework and are captured along with GPU events by Nvidia's nvprof and Nsight profilers. However, this approach only annotates GPU kernel-level information with layer names and lacks the layer-level profiling reported by the framework. 
Moreover, using these instrumented frameworks creates vendor lock-in - making the profiling and analysis dependent on the vendor's frameworks and profilers. This is not an option for ML models developed or deployed using customized or non-vendor supported frameworks.

To address the above issue, we propose XSP - an acrossstack profiling design along with a leveled experimentation methodology. XSP innovatively leverages distributed tracing to aggregate and correlate the profiles from different sources into a single timeline trace. Through the leveled experimentation methodology, XSP copes with the profiling overhead and accurately captures the profiles at each HW/SW stack level Users can use XSP to have a smooth hierarchical step-through of model performance at different levels within the HW/SW stack and identify bottlenecks. Unlike existing approaches, XSP requires no framework modifications. We implement the profiling design for GPUs and couple it with an across-stack analysis pipeline. The analysis pipeline consumes the acrossstack profiling trace and performs 15 types of automated analyses (Table I). These analyses allow us to characterize ML models and their interplay with frameworks, libraries, and hardware. The consistent profiling and automated analysis workflows in XSP enable systematic comparisons of models, frameworks, and hardware.

In summary, this paper makes the following contributions:

- We propose XSP, an across-stack profiling design that innovatively leverages distributed tracing to aggregate profile data from different profiling sources and construct a holistic view of ML model execution.

- We introduce a leveled experimentation methodology that allows XSP to accurately capture the profile at each HW/SW stack level despite the profiling overhead.

- We implement the design for GPU ML model inference and couple it with an analysis pipeline that performs 15 types of automated analyses to systematically characterize ML model execution

- We conduct comprehensive experiments to show the utility of XSP. We use 65 state-of-the-art ML models from MLPerf Inference, AI-Matrix, and TensorFlow and MXNet model zoos. We evaluate the models on 5 representative systems that span the past 4 GPU generations (Turing, Volta, Pascal, and Maxwell) and present performance insights that would otherwise be difficult to discern absent XSP.

The rest of the paper is organized as follows. Section II describes the current profiling tools and benchmarking efforts within the ML and system communities. Section III presents our design and implementation. Section III-D showcases 15 types of automated analysis that can be performed. Section IV further evaluates $65 \mathrm{ML}$ models and presents some insights that are enabled by our design. Section $\mathrm{V}$ concludes this paper.

\section{ML PROFILING ON GPUS AND RELATED WORK}

Researchers leverage different tools and methods to profile ML model execution at each specific level of the HW/SW stack on GPUs. Figure 1 illustrates the model-, layer-, and GPU kernel-level profiling levels on GPUs.
(1) Model-level profiling measures the steps within the model inference pipeline. There exist active efforts by both research and industry to develop benchmark suites [7], [8] to measure and characterize models under different workload scenarios. For model-level profiling, researchers manually insert timing code around inference steps such as input pre-processing, model prediction, and output post-processing. Researchers then use the results as reference points to compare models or systems.

2 Layer-level profiling measures the layers executed by the ML framework using the framework's profilers [1], [2]. These framework profilers are either built-in to the framework or are community-contributed framework plugins. The layer index, name, latency, and memory allocations are captured by the framework profiler as it is executing the layers. Researchers explicitly enable the framework's profiler in their code to get the layer-level profile in a framework-specific format.

3 GPU kernel-level profiling measures the low-level GPU information. Using NVIDIA's nvprof and Nsight profilers, researchers capture the executed GPU kernels information such as their name, latency and metrics. NVIDIA's nvprof and Nsight profilers are built on top of the NVIDIA CUPTI library [9], which provides an API to capture CUDA API, GPU kernel, and GPU metric information.

The disconnect between the above profiling levels prohibits researchers from being able to have a holistic view of model execution - thus, limiting the types of analysis which can be performed. Take the MLPerf_ResNet50_v1.5 model in Figure 1 for example. One can use the aforementioned profiling tools to get the most time-consuming layer (the $208^{\text {th }}$ layer which is named conv2d_48/Conv2D) and the most time-consuming GPU kernel (volta_scudnn_128x64_relu_interior_nn_v1). However, because of the lack of correlation between the GPU kernels and the layers, no other useful analysis can be performed. E.g, one cannot figure out the GPU kernels invoked by the most time-consuming layer, or correlate the most time-consuming GPU kernel to a specific layer within the model. Knowing the correlation between layers and GPU kernels enables more meaningful analyses and informs more optimization opportunities.

Currently, other than modifying framework source code, no tool or method exists to correlate the GPU kernel-level profile to the layer-level profile. For example, to be able to correlate GPU kernels to a certain layer, researchers manually instrument the framework's source code with NVTX markers to annotate layers [10]. The NVTX markers are captured by the nvprof or Nsight profilers and kernels within the markers' ranges belong to the annotated layers. Since the correlation between GPU kernels and layers is highly desired, NVIDIA provides modified versions of frameworks as Docker containers (NGC) where the frameworks are already instrumented with NVTX markers. While the profile captured in this approach correlates GPU kernels with layers, it lacks critical layer-level profiling (such as memory allocations performed by a framework for a layer). Furthermore, current implementations [10] introduce barriers which inhibit frameworks from performing certain optimizations (such as layer-fusion) since the NVTX layer 
marking is performed by surrounding each layer with a "start NVTX marker" layer and an "end NVTX marker" layer. Finally, using vendor frameworks is not an option for profiling ML models developed with customized frameworks - a common practice when using user-defined layers.

To overcome the unknown correlation between layers and GPU kernels without vendor lock-in, there have been efforts [11], [12] to develop fine-grained micro-benchmarks of representative layers. These micro-benchmarks target convolution or RNN layers and are purposely built for algorithm developers, compiler writers, and system researchers. Using layer parameters of popular models, these micro-benchmark measure each layer in isolation. Thus, they do not reflect how layers are executed by frameworks. At best, micro-benchmarks give a lower-bound estimate of how layers would perform in an ideal scenario. This lower-bound can be used to pinpoint potential optimizations in the HW/SW stack [13]. Recent benchmark suites take a multi-tier approach [8], [14] and provide a collection of benchmarks that cover both end-to-end model and layer benchmarking.

We believe a profiling design which captures ML model executions at different HW/SW stack levels and correlates profile data from the different sources - coupled with automated analyses of the results - would boost the productivity of researchers and help understand the model/system performance and identify the bottlenecks. The authors are unaware of any previous work on the aforementioned across-stack profiling. Hence, we design XSP.

\section{XSP DESIGN AND IMPLEMENTATION}

\section{A. Across-Stack Profiling Through Distributed Tracing}

To incorporate profile data from different sources and to create a holistic hierarchical view of ML model execution, XSP leverages distributed tracing [15]-[17]. This section presents XSP's across-stack profiling design.

Distributed tracing is a technique originally conceived for distributed applications, e.g. the ones built using a microservice architecture. In distributed tracing terminology, a timed operation representing a piece of work is referred to as a span. Each span contains a unique identifier (used as its reference), start/end timestamps, and user-defined annotations such as name, key-value tags, and logs. A span may also contain a parent reference to establish a parent-child relationship. Each service in a distributed application has a tracer — some code to create and publish spans. Spans are published to a tracing server which is run on a local or remote system. The tracing server aggregates the spans published by the different tracers into one application timeline trace.

We observe similarities between distributed tracing and across-stack profiling. Based on this observation, we propose XSP, an across-stack profiling design. Profiling across stack levels can be represented using the distributed tracing terminology by: (1) each profiler within a stack is turned into a tracer, (2) the profiled events each form a span, (3)each span is tagged with its stack level, and (4) the parent-child relationship is encoded using a parent reference. The conversion from the profiled events to spans can be performed online while the profiler is running, or can be performed off-line by processing the output of the profiler. The published spans across the stack levels are aggregated by a tracing server into a single timeline trace. Multiple tracers (or profilers) can exist within a stack level, e.g. both CPU and GPU tracers can co-exist at system library or hardware level. As a feature supported by distributed tracing, tracers can be enabled or disabled at runtime.

During span creation, we can, in some cases, associate it with a parent (e.g. map the layer-level spans to the model prediction span). In other cases, because of the use of disjoint profilers, manually associating the child span with its immediate parent is not possible (e.g. map the GPU kernel-level spans to the layer-level spans). To reconstruct the missing parentchild relationship of the profiled events captured by different profilers, XSP's profile analysis builds an interval tree [18] and populates it with intervals corresponding to the spans' start/end timestamps. Using the interval tree, XSP reconstructs the parentchild relationship by checking for interval set inclusion (if the interval span $s_{1}$ contains the interval span $s_{2}$ and the level of $s_{1}$ is one level higher than the level of $s_{2}$, then $s_{1}$ is a parent of $s_{2}$ ). It is possible that there are parallel events where it may be ambiguous to determine a span's parent. In those cases, XSP requires another profiling run where the parallel events are serialized to get the missing correlation information. This can be performed by specifying environment variables without modifications to the application - e.g. setting either CUDA_LAUNCH_BLOCKING=1 for GPUs using CUDA or OMP_NUM_THREADS=1 for CPUs using OpenMP.

To profile asynchronous functions, XSP captures two spans for each asynchronous function denoting their asynchronous launch (called a launch span) and future execution (called an execution span). XSP correlates the two spans using a correlation identifier which is inserted as a span tag during span creation. XSP uses the launch span's parent as the parent of the asynchronous function and uses the execution span to get the performance information or find child spans. E.g., to profile asynchronous GPU kernels, XSP captures both the kernel launch and execution spans (as detailed in Section III-B).

\section{B. Across-stack Profiling on GPUs}

While the across-stack profiling design presented above is general, this paper focuses on the profiling of ML models on GPUs across the model, layer, and GPU kernel level:

(1) Model-level profiling - To profile at the model granularity, XSP provides tracing APIs - startspan and finishSpan - which can be placed within the inference code to measure code regions of interest. For example, to measure the time spent running the model prediction using the framework C APIs, one places the tracing APIs around the calls to TF_SessionRun for TensorFlow or MXPredForward for MXNet. This only requires adding two extra lines in the user's inference code.

2Layer-level profiling - To profile at the layer granularity, XSP uses the ML framework's existing profiling capability. 
M: Model-level Profiling L: Layer-level Profiling G: GPU Kernel-level Profiling

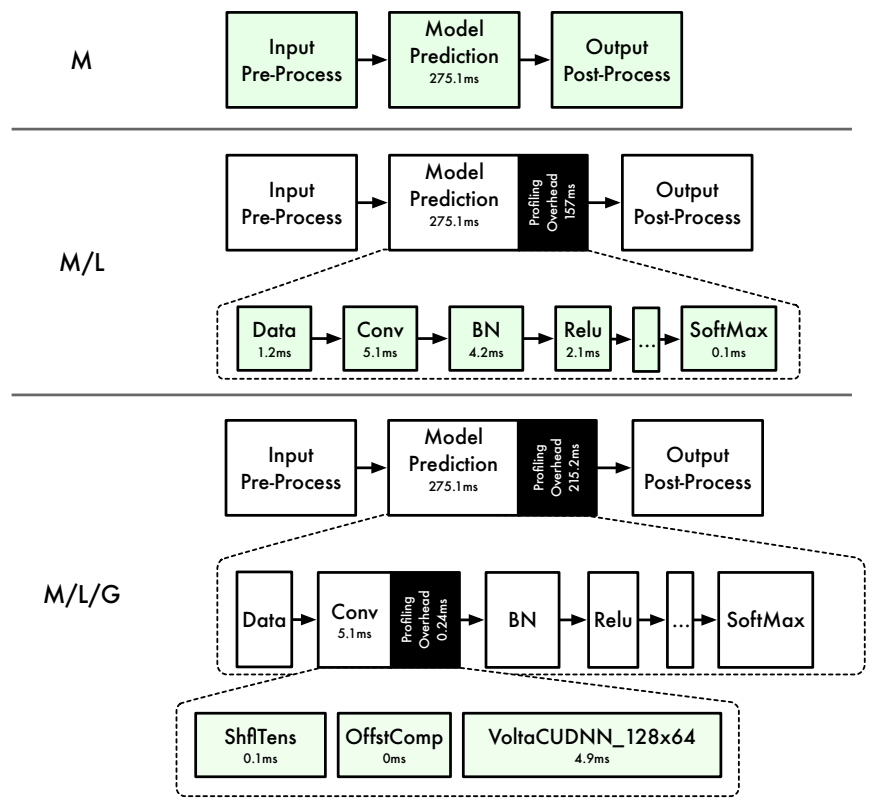

Fig. 2. XSP profiles for MLPerf_ResNet50_v1.5 with batch size 256 on Tesla_V100 (Table VI) with the model-level (M), model-/layer-level $(\mathrm{M} / \mathrm{L})$, and model-/layer-/GPU kernel-level (M/L/G) profiling. At each level, the green components correctly measure the latency whereas the rest incur profiling overhead.

During runtime, XSP enables the framework profiler, converts the profile results into spans, and publishes them to the tracing server. In TensorFlow, enabling layer profiling requires calling the framework's prediction function with the profiling option enabled. This option is controlled by the RunOptions.TraceLevel setting which is passed to the TF_SessionRun function in TensorFlow. In MXNet, the MXSetProfilerState function enables and disables layer profiling. Similar mechanisms exist for other frameworks such as Caffe, Caffe2, PyTorch, and TensorRT. The layer spans are set to be the children of the model prediction span, and hence each layer are directly correlated to the model prediction step. Since XSP leverages the existing framework's profiling capabilities, profiling at the layer level require no modification to the framework's source code.

3GPU kernel-level profiling - To obtain the GPU profile, XSP uses NVIDIA's CUPTI library [9]. The CUPTI library captures the CUDA API calls, GPU activities (GPU tasks such as kernel executions and memory copies), and GPU kernel metrics (low-level hardware counters such as GPU achieved occupancy, flop count, and memory read/write for GPU kernels). Similar to Nsight or nvprof (which are built on top of CUPTI), one can specify with XSP which CUDA APIs, GPU activities, or metrics to capture. At runtime, XSP converts the captured CUPTI information into spans and publishes them to the tracer server (asynchronously to avoid added overhead). If profiling GPU metrics is enabled, the metrics are added as metadata to the corresponding kernel's span.

GPU kernels are often launched asynchronously by the ML frameworks or libraries. Therefore, for each kernel two spans are created within the XSP timeline. XSP uses the CUPTI callback API to capture the CUDA API call cudaLaunchKernel as the launch span. XSP uses the CUPTI activity API to capture the effective kernel duration as the execution span. XSP uses the kernel launch span to associate it with the parent layer span and use the execution span to get the kernel performance information. The two spans are correlated by the correlation_id provided by CUPTI. Since this correlation can potentially be expensive, we perform correlation during profile analysis which aggregates the information from two GPU kernel spans.

\section{Dealing with Profiling Overhead through Leveled Experi- mentation}

Profiling always comes with overhead. We observe that creating spans online adds negligible overhead per span (and no overhead exists if the profile is converted offline). Thus, XSP incurs only the profiling overhead introduced by the integrated profilers. For example, layer-level profiling adds overhead to the model prediction depending on how many layers are executed. And as with the existing NVIDIA profilers, the GPU-level profiling incurs overhead, which can be substantial depending on if GPU metric profiling is enabled and the types of GPU metrics to capture. GPU memory metrics are especially expensive to profile and can slow down execution by over $100 \times$. This is due to the limited number of GPU hardware performance counters, which require GPU kernels to be replayed multiple times to capture the user-specified metrics.

Profilers at a specific stack level accurately capture the events within that level. And, since tracers in XSP can be enabled or disabled depending on the characterization target, the profiling overhead can be controlled by picking the profiling level. For an event at level $n$ (where level 1 is the model level), the profiling overhead introduced at level $n+1$ can be quantified by subtracting the latency of the event when profilers up to level $n$ are enabled from the latency when profilers up to level $n+1$ are enabled. We refer to the profiling practice which uses traces from multiple runs with different profiling levels as leveled experimentation. Through leveled experimentation, XSP gets accurate timing of profiled events at all stack levels.

To demonstrate the profiling overhead and the leveled experimentation, we use the MLPerf_ResNet50_v1.5 model running on the Tesla_V100 system (Table VII) as an example. Figure 2 shows the models XSP profiles at different profiling levels. We can enable the model-level profiling (M) to get the baseline model prediction latency of $275.1 \mathrm{~ms}$. To further measure the latency of each layer, we enable both the model- and layer-level profiling (M/L). While the layer-level profiling adds overhead to the model prediction latency, it accurately captures the latency of each layer. We can quantify this overhead by subtracting the model prediction latency in the model-level profile from the model prediction latency in the model-/layer-level profile. We find that the layer-level profiling introduces a $157 \mathrm{~ms}$ overhead. We can further perform the GPU kernel-level profiling along with the model-/layer-level profiling to get a hierarchical view of the model execution 
TABLE I

THE 15 ANALYSES PERFORMED BY XSP. THE ANALYSES REQUIRE PROFILING INFORMATION FROM ONE OR MORE LEVELS (M: MODEL-LEVEL, L: LAYER-LEVEL, AND G: GPU KERNEL-LEVEL).

\begin{tabular}{|c|c|c|c|c|c|c|}
\hline & Analysis & $\begin{array}{c}\text { Profiling } \\
\text { Levels }\end{array}$ & $\begin{array}{c}\begin{array}{c}\text { End-to-End } \\
\text { Benchmarking }\end{array} \\
\end{array}$ & $\begin{array}{c}\text { Framework } \\
\text { Profilers }\end{array}$ & $\begin{array}{l}\text { NVIDIA } \\
\text { Profilers }\end{array}$ & XSP \\
\hline A1 & Model information table & $\mathbf{M}$ & 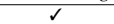 & $x$ & $x$ & $\mathrm{a}$ \\
\hline A2 & Layer information table & $\mathbf{L}$ & $x$ & $\checkmark$ & $x$ & $\checkmark$ \\
\hline$\overline{\mathrm{A3}}$ & Layer latency & $\mathbf{L}$ & $x$ & $\checkmark$ & $x$ & $\checkmark$ \\
\hline A4 & Layer memory allocation & $\mathrm{L}$ & $x$ & $\checkmark$ & $x$ & $\checkmark$ \\
\hline A5 & Layer type distribution & $\mathbf{L}$ & $x$ & $\checkmark$ & $x$ & $\checkmark$ \\
\hline A6 & Layer latency aggregated by type & $\mathbf{L}$ & $x$ & $\checkmark$ & $x$ & $\checkmark$ \\
\hline A7 & Layer memory allocation aggregated by type & $\mathbf{L}$ & $x$ & $\checkmark$ & $x$ & $\checkmark$ \\
\hline A8 & GPU kernel information table & G & $x$ & $x$ & $\checkmark$ & $\checkmark$ \\
\hline A9 & GPU kernel roofline & G & $x$ & $x$ & 2 & $\checkmark$ \\
\hline A10 & GPU kernel information aggregated by name table & G & $x$ & $x$ & $\checkmark$ & $\checkmark$ \\
\hline A11 & GPU kernel information aggregated by layer table & L/G & $x$ & $x$ & $x$ & $\checkmark$ \\
\hline A12 & GPU metrics aggregated by layer & $\mathbf{L} / \mathbf{G}$ & $x$ & $x$ & $x$ & 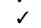 \\
\hline A13 & GPU vs Non-GPU latency & $\mathbf{L} / \mathbf{G}$ & $x$ & $x$ & $x$ & $\checkmark$ \\
\hline A14 & Layer roofline & L/G & $x$ & $x$ & $x$ & $\checkmark$ \\
\hline A15 & GPU kernel information aggregated by model table & $\mathbf{M} / \mathbf{G}$ & $x$ & $x$ & s & 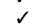 \\
\hline
\end{tabular}

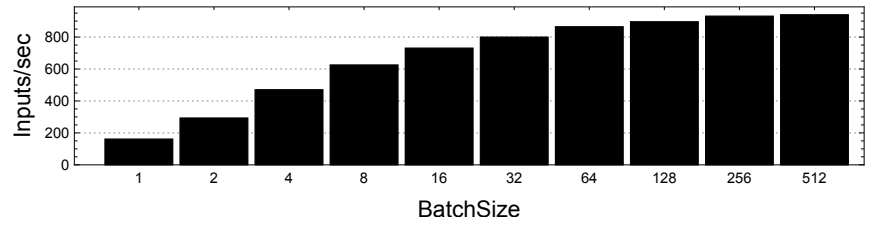

Fig. 3. The throughput of MLPerf_ResNet50_v1.5 across batch sizes on Tesla_V100.

$(\mathrm{M} / \mathrm{L} / \mathrm{G})$. Enabling the GPU kernel-level profiling adds extra overhead to the model prediction latency — making the model prediction step (with the added overhead) take $490.3 \mathrm{~ms}$. If we look at the first convolution layer, the GPU profiling of the 3 child kernels incurs a $0.24 m s$ overhead. We verified the layer and GPU kernel latencies measured by XSP against what framework and NVIDIAs profilers report.

\section{Across-Stack Analysis}

We couple XSP with an automated analysis pipeline which consumes the profiling traces published to the tracing server. We define 15 analyses that capture across-stack characteristics of ML model executions on GPUs as listed in Table I. The 15 analyses are grouped into 3 categories based on the profiling information required. Since meaningful characterization requires multiple runs, the pipeline takes traces from a userdefined number of evaluations, correlates the information, and computes the trimmed mean value (or other user-defined statistical summaries) for the same performance value (e.g. latency) across runs. This automated analysis pipeline allows users to systematically and efficiently characterize and compare ML models.

To illustrate the analyses, we use the TensorFlow MLPerf_ResNet50_v1.5 model (ID $=7$ in Table VIII) from the MLPerf Inference v0.5 release. The model is run within the NGC TensorFlow container v19.06 on an AWS P3 [19] instance (Tesla_V100 in Table VII). The P3 instance is equipped with a Tesla V100-SXM2 GPU and achieves a peak throughput of 15.7 TFlops and $900 \mathrm{~GB} / \mathrm{s}$ global memory bandwidth. Batch size 256 is used in Sections III-D2 and III-D3, since the model achieves maximum throughput at that batch size. Using XSP, one can perform analyses that are either difficult or impossible using existing tools or methods.
TABLE II

THE TOP 5 MOST TIME CONSUMING LAYERS IN A2 FOR MLPERF_RESNET50_V1.5 WITH BATCH SIZE 256 ON TESLA_V100. IN TOTAL, THERE ARE 234 LAYERS OF WHICH 143 TAKE LESS THAN 1 MS.

\begin{tabular}{rlcccr}
\hline $\begin{array}{c}\text { Layer } \\
\text { Index }\end{array}$ & $\begin{array}{l}\text { Layer } \\
\text { Name }\end{array}$ & $\begin{array}{c}\text { Layer } \\
\text { Type }\end{array}$ & $\begin{array}{c}\text { Layer } \\
\text { Shape }\end{array}$ & $\begin{array}{c}\text { Latency } \\
(\mathbf{m s})\end{array}$ & $\begin{array}{c}\text { Alloc Mem } \\
(\mathbf{M B})\end{array}$ \\
\hline 208 & conv2d_48/Conv2D & Conv2D & $\langle 256,512,7,7\rangle$ & 7.59 & 25.7 \\
221 & conv2d_51/Conv2D & Conv2D & $\langle 256,512,7,7\rangle$ & 7.57 & 25.7 \\
195 & conv2d_45/Conv2D & Conv2D & $\langle 256,512,7,7\rangle$ & 5.67 & 25.7 \\
3 & conv2d/Conv2D & Conv2D & $\langle 256,64,112,112\rangle$ & 5.08 & 822.1 \\
113 & conv2d_26/Conv2D & Conv2D & $\langle 256,256,14,14\rangle$ & 4.67 & 51.4 \\
\hline
\end{tabular}

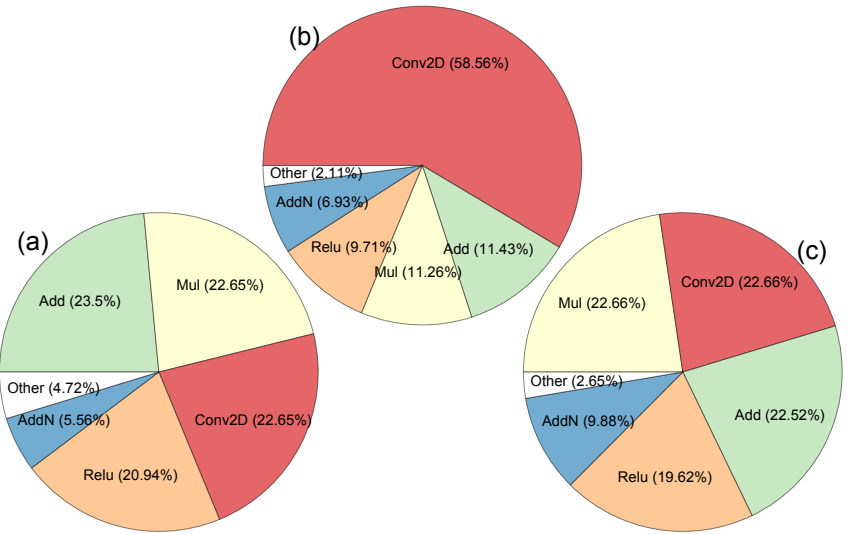

Fig. 4. Layer statistics for MLPerf_ResNet50_v1.5 on Tesla_V100: (a) A5 layer type distribution, (b) $\mathbf{A 6}$ layer latency aggregated by type, (c) A7 layer memory allocation aggregated by type.

1) Using Model-level Profile: Both model throughput and latency are important to researchers who want to understand a model's end-to-end performance. Using only the modellevel profiling, XSP automates the computation of a model's throughput and latency across batch sizes and generate a A1 model information table. XSP then computes the model's optimal batch size given a user-defined metric (e.g. a latency target). By default XSP computes the optimal batch size by evaluating the model across batch sizes and selecting the batch size where doubling it does not increase the model's throughput by more than $5 \%$. Figure 3 shows the throughput of MLPerf_ ResNet50_v1.5 across batch sizes. XSP computes the optimal batch size as 256 where the model achieves a maximum throughput of 930.7 images/second. The corresponding batch latency is $275.05 \mathrm{~ms}$. Absent XSP, researchers insert timing functions around the model prediction code, perform multiple evaluations, and write scripts to compute the model's throughput, latency, and optimal batch size.

2) Using Model- and Layer-level Profiles: Using both the model- and layer-level profiles enables characterization of layers executed by the ML framework. The measured layers may be different from the ones statically defined in the model graph, since a framework may perform model optimization at runtime. Using the data captured, XSP generates a A2 layer information table reporting index, name, shape, latency, and allocated memory of all the layers. For example, Table II shows the top 5 most time-consuming layers for MLPerf_ResNet50_v1.5.

XSP further uses the profile data to visualize both the 

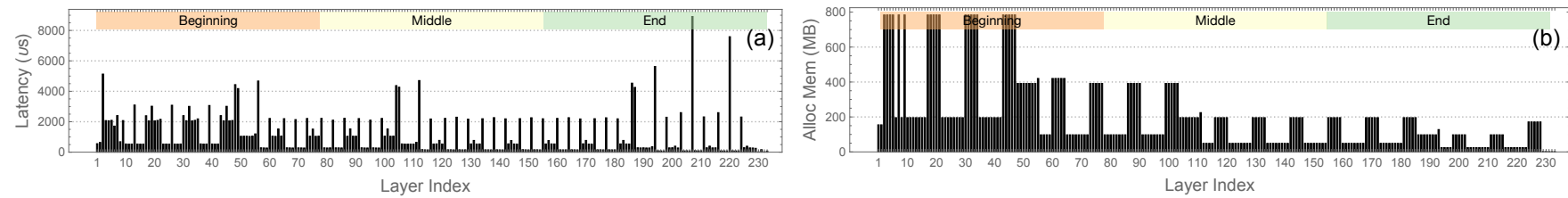

Fig. 5. The (a) A3 latency and (b) A4 memory allocation for each layer in MLPerf_ResNet50_v1.5 with batch size 256 on Tesla_V100. To understand the performance trend, we divide the model execution into 3 intervals based on the layer index: beginning, middle, and end.

A3 latency per layer and A4 allocated memory per layer in layer execution order. Figures 5 shows the two analyses for MLPerf_ResNet50_v1.5 at the optimal batch size. We observe that a layer latency and memory allocation trend exists - the model latency can be mostly attributed to the early executed layers. Similarly, the memory allocation is high for the early stage of the model execution, and less so during the middle and end stages.d

We can group the layer information by layer type to derive useful layer execution statistics such as $\mathbf{A} 5$ the number of times each layer type is executed (Figure $4 a$ ), the $\mathbf{A 6}$ layer latency aggregated by type (Figure 4b), and the A7 layer memory allocation aggregated by type (Figure $4 \mathrm{c}$ ). We observe that MLPerf_ResNet50_v1.5 mostly comprises of Add, Conv2D, Mul, and Relu layers. This is because of the ResNet modules which have the pattern of Conv $\rightarrow \mathrm{BN} \rightarrow$ Relu. The ResNet modules get executed by TensorFlow as a Conv2D $\rightarrow \mathrm{Mul} \rightarrow \mathrm{Add} \rightarrow \mathrm{Relu}$ layer sequence. This same group of layers dominates both latency and memory allocation, with Conv2D being the most time-consuming layer type.

Absent XSP, researchers use the framework profiler to gather layer-level information. Through manually parsing and aggregating the profiling output across runs, researchers can perform A2-7. However, since the output format of a framework profiler is framework-dependent, the analysis scripts developed in this case are also framework-specific.

3) Using Model-, Layer-, and GPU Kernel-level Profiles: To distill fine-grained performance information, XSP uses model-, layer- and GPU kernel-level profiles to generate a A8 GPU kernel information table summarizing all the kernels in the model prediction. An example is shown in Table III where the top 5 most time consuming GPU kernel calls for MLPerf_ResNet50_v1.5 are listed. The 5 kernels perform either matrix multiplication or convolution. All the GPU metrics supported by the NVIDIA profiling tools [20] can be captured through XSP, here we focus on flop_count_sp, dram_read_bytes, dram_write_bytes, and achieved_occupancy:

- flop_count_sp - the total number of single-precision floating-point operations executed by a kernel.

- dram_read_bytes - the total number of bytes read from the GPU's DRAM to its L2 cache in a kernel.

- dram_write_bytes - the total number of bytes written from the GPU's L2 cache to its DRAM in a kernel.

- achieved_occupancy - the ratio of the average active warps per active cycle to the maximum number of warps per streaming multiprocessor. The achieved_occupancy is an indicator to the level of parallelism for a kernel.

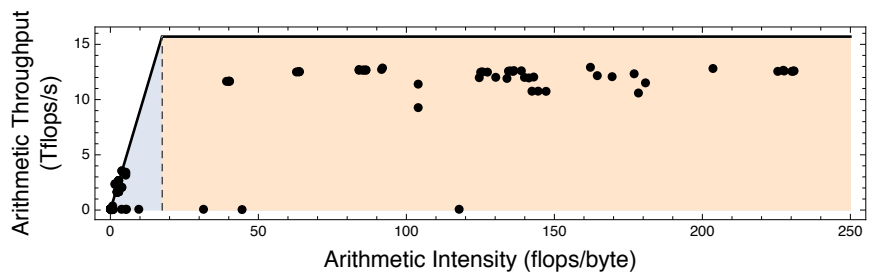

Fig. 6. The A9 roofline analysis for the GPU kernels in MLPerf_ResNet50_v1.5 with batch size 256 on Tesla_V100. Kernels within the blue region are memory-bound, whereas the ones within the orange region are compute-bound.

Using both the kernel flop and memory access metrics, XSP calculates the kernel arithmetic intensity and arithmetic throughput. These parameters are used to perform GPU kernel roofline [21] analysis. A kernel's arithmetic intensity is the ratio between the number of flops and the number of memory accesses: arithmetic_intensity $=\frac{\text { flop_count_sp }}{\text { dram_read_bytes+dram_write_bytes }}$. A kernel's arithmetic throughput is the ratio between the number of flops and the latency: arithmetic_throughput $=\frac{\text { flop_count_sp }}{\text { kernel latency }}$. Using the GPU's theoretical FLOPS and memory bandwidth, we compute the ideal arithmetic intensity using the equation: ideal_arithmetic_intensity $=\frac{\text { peak_FLOPS }}{\text { memory_bandwidth }}$. The Tesla_V100 GPU, for example, has a peak throughput of 15.7 TFLOPS and a global memory bandwidth of $900 \mathrm{~GB} / \mathrm{s}$, hence an ideal arithmetic intensity of $\frac{15.7 \mathrm{TFLOPS}}{900 \mathrm{~GB} / \mathrm{s}}=17.44$ flops/byte. A kernel is memory-bound if its arithmetic intensity is less than the GPU's ideal arithmetic intensity (blue region) and is compute-bound otherwise (orange region). A9 visualizes the roofline analysis of all the GPU kernels (shown in Figure 6). As expected, the most time-consuming kernels are convolution kernels which are all compute-bound.

XSP creates a table of A10 GPU kernel information aggregated by name, as shown in Table IV. The aggregated kernel latency, flops, and DRAM reads and writes are calculated as the sum of all the kernel instances with the same name. The aggregated kernel achieved occupancy is calculated as the weighted sum (by kernel latency) of achieved occupancy of all the kernel instances with the same name. The aggregated kernel arithmetic intensity and throughput are calculated using the aggregated flops and memory accesses. For MLPerf_ResNet50_v1.5, we observe that the most time consuming GPU kernel is volta_scudnn_128 124 -relu_interior_nn_v1 from the cuDNN [22] library, which is compute-bound and takes $30.87 \%$ of the overall model prediction latency. The $2^{\text {nd }}$ and $3^{\text {rd }}$ most time consuming kernels 
TABLE III

THE TOP 5 MOST TIME-CONSUMING KERNELS IN A8 FOR MLPERF_RESNET50_V1.5 ON TeS LA_V100. IN TOTAL, 375 KERNELS ARE INVOKED OF WHICH 284 TAKE LESS THAN $1 m s$.

\begin{tabular}{|c|c|c|c|c|c|c|c|c|c|}
\hline Kernel Name & $\begin{array}{l}\text { Layer } \\
\text { Index }\end{array}$ & $\begin{array}{c}\text { Layer } \\
\text { Kernel } \\
\text { Latency } \\
(\mathbf{m s})\end{array}$ & $\begin{array}{l}\text { Kernel } \\
\text { Gflops }\end{array}$ & $\begin{array}{c}\text { Kernel } \\
\text { DRAM } \\
\text { Reads } \\
(\text { MB })\end{array}$ & $\begin{array}{l}\text { Kernel } \\
\text { DRAM } \\
\text { Writes } \\
\text { (MB) }\end{array}$ & $\begin{array}{c}\text { Kernel } \\
\text { Achieved } \\
\text { Occupancy } \\
(\%)\end{array}$ & $\begin{array}{c}\text { Kernel } \\
\text { Arithmetic } \\
\text { Intensity } \\
\text { (flops/byte) }\end{array}$ & $\begin{array}{c}\text { Kernel } \\
\text { Arithmetic } \\
\text { Throughput } \\
\text { (Tflops/s) }\end{array}$ & $\begin{array}{l}\text { Memory } \\
\text { Bound? }\end{array}$ \\
\hline volta_cgemm_32×32_tn & 221 & 6.04 & 77.42 & 40.33 & 43.86 & 12.18 & 876.97 & 12.82 & $x$ \\
\hline volta_cgemm_32×32_tn & 208 & 6.03 & 77.42 & 43.93 & 43.81 & 12.19 & 841.59 & 12.83 & $x$ \\
\hline volta_scudnn_128x128_relu_interior_nn_v1 & 195 & 5.48 & 59.20 & 27.71 & 8.40 & 15.49 & $1,563.30$ & 10.80 & $x$ \\
\hline volta_scudnn_128x64_relu_interior_nn_v1 & 3 & 4.91 & 62.89 & 11.55 & 283.05 & 13.20 & 203.58 & 12.81 & $x$ \\
\hline volta_scudnn_128×128_relu_interior_nn_v1 & 57 & 4.56 & 59.24 & 34.83 & 37.64 & 15.15 & 779.55 & 12.99 & $x$ \\
\hline
\end{tabular}

TABLE IV

THE TOP 5 MOST TIME-CONSUMING KERNELS IN A10 FOR MLPERf_RESNET50_V1. 5 ON TEs LA_V100. 30 UNIQUE KERNELS ARE INVOKED IN TOTAL.

\begin{tabular}{|c|c|c|c|c|c|c|c|c|c|c|}
\hline Kernel Name & $\begin{array}{l}\text { Kernel } \\
\text { Count }\end{array}$ & $\begin{array}{c}\text { Kernel } \\
\text { Latency } \\
(\mathbf{m s})\end{array}$ & $\begin{array}{c}\text { Kernel } \\
\text { Latency } \\
\text { Percentage }\end{array}$ & $\begin{array}{l}\text { Kernel } \\
\text { Gflops }\end{array}$ & $\begin{array}{c}\text { Kernel } \\
\text { DRAM } \\
\text { Reads } \\
\text { (MB) }\end{array}$ & $\begin{array}{c}\text { Kernel } \\
\text { DRAM } \\
\text { Writes } \\
\text { (MB) }\end{array}$ & $\begin{array}{c}\text { Kernel } \\
\text { Achieved } \\
\text { Occupancy } \\
(\%)\end{array}$ & $\begin{array}{c}\text { Kernel } \\
\text { Arithmetic } \\
\text { Intensity } \\
\text { (flops/byte) }\end{array}$ & $\begin{array}{c}\text { Kernel } \\
\text { Arithmetic } \\
\text { Throughput } \\
\text { (Tflops/s) }\end{array}$ & $\begin{array}{c}\text { Memory } \\
\text { Bound? }\end{array}$ \\
\hline volta_scudnn_128x64_relu_interior_nn_v1 & 34 & 84.95 & 30.87 & $1,053.63$ & $4,429.64$ & $5,494.22$ & 22.58 & 101.25 & 12,40 & $x$ \\
\hline Eigen: :TensorCwiseBinaryOp<scalar_product_op $>$ & 52 & 28.43 & 10.33 & 2.85 & $4,181.23$ & $6,371.12$ & 49.72 & 0.26 & 0.10 & $\checkmark$ \\
\hline Eigen: :TensorCwiseBinaryop<scalar_sum_op $>$ & 51 & 26.38 & 9.59 & 2.64 & $4,063.49$ & $6,052.22$ & 49.69 & 0.25 & 0.10 & $\checkmark$ \\
\hline Eigen: :TensorCwiseBinaryop $<$ scalar_max_op $>$ & 48 & 24.71 & 8.98 & 0 & $3,773.84$ & $5,699.95$ & 98.39 & 0 & 0 & $\checkmark$ \\
\hline volta_scudnn_128x128_relu_interior_nn_v1 & 4 & 23.02 & 8.37 & 276.64 & 671.68 & 335.01 & 15.96 & 262.08 & 12,02 & $x$ \\
\hline
\end{tabular}

are scalar_product_op and scalar_sum_op and are defined by the Eigen [23] library, are memory-bound, and take $10.33 \%$ and $9.59 \%$ of the model inference latency, respectively.

Since each GPU kernel can be correlated to the layer that invokes it, XSP aggregates the information of GPU kernels within each layer and builds a table of A11 GPU kernel information aggregated by layer. A layer's kernel latency, flops, DRAM reads and writes are calculated by adding the corresponding values of all the kernels invoked by that layer. The layer's achieved occupancy is calculated as the weighted sum (by kernel latency) of the achieved occupancy of all the kernels within the layer. As an example, Table V shows the aggregated GPU kernel information for the top 5 most timeconsuming layers in MLPerf_ResNet50_v1.5.

Using this data, XSP visualizes the A12 total flops, DRAM reads and writes per layer (shown in Figure 7 (a), (b) and (c) respectively). Subtracting a layer's total GPU kernel latency from the its overall latency computes the A13 time not spent performing GPU computation. We call this difference the layer's non-GPU latency. Figure 8 shows the layer's GPU and non-GPU latency normalized to the overall layer latency for MLPerf_ResNet50_v1.5. The layer arithmetic intensity and throughput are calculated using the layer's total flops and memory accesses. A A14 roofline analysis of all the layers is performed in Figure 9. We observe that the Conv2D layers are the most compute and memory intensive. The Conv2D, MatMul, BiasAdd, and Softmax layers are compute-bound, whereas the other layers (Add, Mul, and Relu) are memorybound.

XSP aggregates all the GPU kernel information within a model and computes a table of the A15 total GPU kernel latency, flop, and memory access information for the model (shown in Table VI). Similar to the layer aggregation, the model kernel latency, flops, DRAM reads and writes are calculated as the sum of all kernels invoked by the model. XSP computes the model's achieved occupancy as the weighted sum (by kernel latency) of the achieved occupancy of all the kernels invoked. The model's arithmetic intensity and throughput are calculated using the model's total flops and memory accesses. This information is used to classify the entire model as either compute- or memory-bound.

Figure 10 visualizes the roofline analysis for MLPerf_ResNet50_v1.5 across batch sizes on Tesla_V100. We see that the model is compute-bound except for batch sizes 16 and 32 where it is memory-bound. Looking into the data in $A 2,8,10$ we find that the kernels invoked for the convolution layers sometimes vary across batch sizes. This is because the cuDNN library relies on heuristics to choose the algorithm used for a convolution layer. The heuristics depend on the layer input parameters, available memory, etc. For batch sizes less than 16, the cuDNN convolution API uses the IMPLICIT_GEMM algorithm and invokes the GPU kernel cudnn: :detail: : implicit_convolve_ sgemm. This kernel has high arithmetic intensity and dominates the model's latency. For batch sizes greater than 16, the cuDNN convolution API chooses a different algorithm - IMPLICIT_ PRECOMP_GEMM algorithm, which invokes the GPU kernel volta_scudnn_128×64_relu_interior_nn_v1. Although this kernel is compute-bound, for batch sizes less than 64 it has a relatively low arithmetic intensity. Thus, for both batch sizes 16 and 32, this kernel's arithmetic intensity is not high enough to compensate for the effects of the other memory-bound kernels. The result is that the overall model is memory-bound for batch sizes 16 and 32 . We also observe that the overall GPU achieved occupancy for the model increases as the batch size approaches the optimal batch size.

A8 and A10 are currently the most common types of analyses performed by researchers using NVIDIA's profilers. Less common, but still possible, analyses without XSP are roofline analyses $\mathrm{A9}$ and $\mathbf{A} 15$ as they require non-trivial 

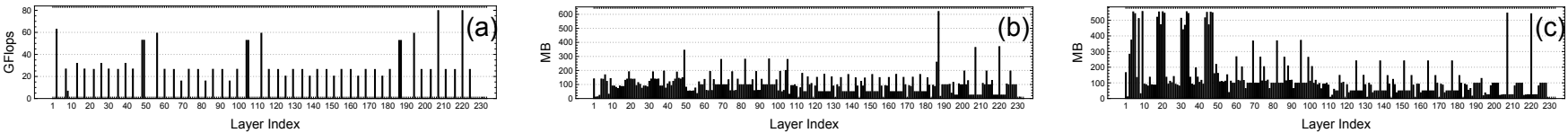

Fig. 7. The A12 total GPU kernel (a) flops, (b) DRAM reads, and (c) DRAM writes per layer for MLPerf_ResNet50_v1.5 with batch size 256 on Tesla_V100.

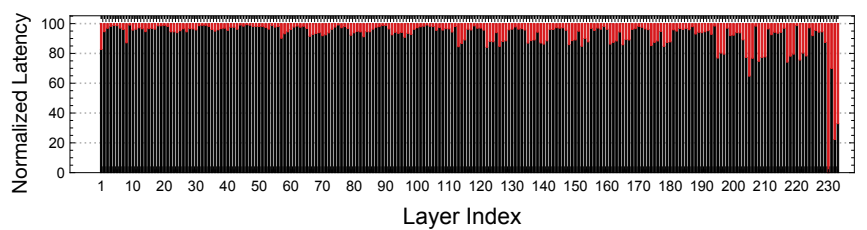

Fig. 8. The A13 normalized GPU and Non-GPU latency per layer for MLPerf_ResNet50_v1.5 with batch size 256 on Tesla_V100.

TABLE V

THE TOP 5 MOST TIME-CONSUMING LAYERS IN A11 FOR MLPERF_RESNET50_V1.5 ON TESLA_V100.

\begin{tabular}{|c|c|c|c|c|c|c|c|c|c|}
\hline $\begin{array}{l}\text { Layer } \\
\text { Index } \\
\end{array}$ & $\begin{array}{c}\text { Layer } \\
\text { Latency } \\
(\mathbf{m s})\end{array}$ & $\begin{array}{c}\text { Kernel } \\
\text { Latency } \\
(\mathrm{ms})\end{array}$ & $\begin{array}{l}\text { Layer } \\
\text { Gflops }\end{array}$ & $\begin{array}{l}\text { Layer } \\
\text { DRAM } \\
\text { Reads } \\
(\text { MB }) \\
\end{array}$ & $\begin{array}{c}\text { Layer } \\
\text { DRAM } \\
\text { Writes } \\
\text { (MB) } \\
\end{array}$ & $\begin{array}{c}\text { Layer } \\
\text { Achieved } \\
\text { Occupancy } \\
(\%)\end{array}$ & $\begin{array}{c}\text { Layer } \\
\text { Arithmetic } \\
\text { Intensity } \\
\text { (flops/byte) }\end{array}$ & $\begin{array}{c}\text { Layer } \\
\text { Arithmetic } \\
\text { Throughput } \\
\text { (Tflops/s) }\end{array}$ & $\begin{array}{l}\text { Memory } \\
\text { Bound? }\end{array}$ \\
\hline 208 & 7.59 & 7.45 & 79.74 & 362.67 & 548.50 & 19.43 & 83.46 & 10.70 & $x$ \\
\hline 221 & 7.57 & 7.43 & 79.74 & 368.11 & 551.70 & 19.43 & 82.68 & 10.73 & $x$ \\
\hline 195 & 5.67 & 5.55 & 59.20 & 36.51 & 17.99 & 15.80 & $1,036.10$ & 10.67 & $x$ \\
\hline 3 & 5.08 & 4.91 & 62.89 & 11.55 & 284.21 & 13.23 & 202.78 & 12.80 & $x$ \\
\hline 113 & 4.67 & 4.57 & 59.22 & 76.65 & 21.36 & 15.31 & 576.17 & 12.94 & $x$ \\
\hline
\end{tabular}

scripts. The scripts parse and aggregate the GPU profilers' outputs across multiple model evaluations to compute the roofline model. Analyses A11-14 cannot be performed using existing tools as they require both the layer- and GPU kernellevel profiles and their results to be correlated.

\section{E. Extensibility}

Care was taken to ensure that XSP's design is extensible. Other profiling tools or methods can be integrated into XSP by implementing XSP's tracer interface. Thus, XSP can be extended with more tracers at each stack level or extended to capture more stack levels. For example, one can integrate CPU profilers into XSP to capture both CPU and GPU information within the same timeline. One can also add a ML library profiling level between the layer- and GPU kernel-level to measure the cuDNN API calls. Adding an application profiling level above the model level to measure whole applications (possibly distributed and using more than one ML model) is naturally supported by XSP as it uses distributed tracing. As new profilers are introduced into XSP, one can add more types of analyses to the automated analysis pipeline.

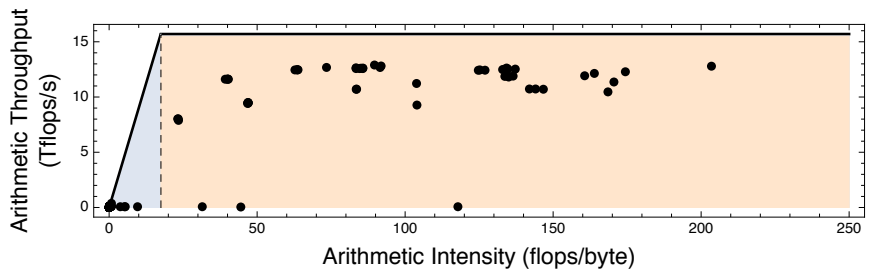

Fig. 9. The A14 roofline analysis for all the layers in MLPerf_ResNet50_v1. 5 with batch size 256 on Tesla_V100.
TABLE VI

THE A15GPU KERNEL INFORMATION AGGREGATED WITHIN MLPERF_RESNET50_V1.5 ACROSS BATCH SIZES ON TESLA_V100.

\begin{tabular}{|c|c|c|c|c|c|c|c|}
\hline $\begin{array}{c}\text { Batch } \\
\text { Size }\end{array}$ & $\begin{array}{c}\text { Model } \\
\text { Latency } \\
(\mathrm{ms})\end{array}$ & $\begin{array}{c}\text { Kernel } \\
\text { Latency } \\
(\mathrm{ms})\end{array}$ & $\begin{array}{l}\text { Model } \\
\text { Gflops }\end{array}$ & $\begin{array}{c}\text { Model } \\
\text { DRAM } \\
\text { Reads } \\
\text { (MB) }\end{array}$ & $\begin{array}{c}\text { Model } \\
\text { DRAM } \\
\text { Writes } \\
\text { (MB) }\end{array}$ & $\begin{array}{c}\text { Model } \\
\text { Achieved } \\
\text { Occupancy } \\
(\%)\end{array}$ & $\begin{array}{l}\text { Memory } \\
\text { Bound? }\end{array}$ \\
\hline 1 & 6.21 & 5.01 & 7.94 & 192.49 & 194.16 & 22.65 & $x$ \\
\hline 2 & 6.83 & 5.93 & 16.08 & 290.41 & 354.54 & 22.47 & $x$ \\
\hline 4 & 8.51 & 7.68 & 30.95 & 659.11 & 720.15 & 26.39 & $x$ \\
\hline 8 & 12.80 & 11.60 & 60.66 & $1,676.07$ & $1,496.81$ & 31.97 & $x$ \\
\hline 16 & 21.90 & 20.14 & 118.04 & $3,969.19$ & $3,024.09$ & 35.58 & $\checkmark$ \\
\hline 32 & 40.03 & 37.14 & 232.78 & $7,711.50$ & $5,823.97$ & 38.76 & $\checkmark$ \\
\hline 64 & 74.03 & 67.72 & 429.08 & $10,932.22$ & $9,268.27$ & 43.18 & $x$ \\
\hline 128 & 142.89 & 131.79 & 873.63 & $16,071.32$ & $16,105.40$ & 44.48 & $x$ \\
\hline 256 & 275.05 & 254.25 & $1,742.39$ & $23,185.11$ & $31,095.45$ & 43.15 & $x$ \\
\hline
\end{tabular}

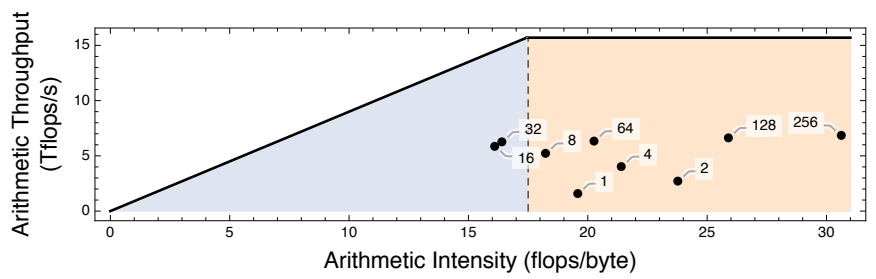

Fig. 10. The roofline analysis for MLPerf_ResNet50_v1.5 across batch sizes on Tesla_V100 using A15.

\section{EVALUATION}

We profile and characterize 55 state-of-the-art TensorFlow ML models (Table VIII) selected from the MLPerf Inference [7], AI-Matrix [8], and TensorFlow model zoo [24]-[26]. The models solve computer vision tasks including image classification, object detection, instance segmentation, semantic segmentation, and super resolution. To compare TensorFlow against MXNet, we select an additional 10 MXNet models from the MXNet Gluon model zoo [27] (Table X) that are comparable to the TensorFlow models. We evaluated the models using NGC TensorFlow container v19.06, and NGC MXNet container v19.06 on 5 representative GPU systems listed in Table VII. This section presents insights about the models, frameworks, and GPU systems using the XSP's analyses described in Section III-D.

\section{A. Model Evaluation}

Using the model- and layer-level profiling data, we look at all 55 TensorFlow models in Table VIII. Models solving the same task are clustered together and are then sorted by their reported accuracy. The table shows each model's accuracy, model graph size, online latency (batch size is 1), maximum throughput, optimal batch size (described in Section III-D1), and percentage of latency attributed to convolution layers.

Model latency percentage of convolution layers - Using the model- and layer-level profile data, we calculate the percentage of model latency attributed to convolution lay- 
TABLE VII

Five systems with Turing, Volta, Pascal, and Maxwell GPUs are Selected For EValuation. We Calculate the ideal arithmetic INTENSITY OF EACH SYSTEM USING THE THEORETIC FLOPS AND MEMORY BANDWIDTH REPORTED BY NVIDIA.

\begin{tabular}{|c|c|c|c|c|c|c|}
\hline Name & CPU & GPU & $\begin{array}{c}\text { GPU } \\
\text { Architecture }\end{array}$ & $\begin{array}{c}\text { Theoretical } \\
\text { FLOPS (TFLOPS) }\end{array}$ & $\begin{array}{c}\text { Memory Bandwidth } \\
\text { (GB/s) }\end{array}$ & $\begin{array}{c}\text { Ideal Arithmetic } \\
\text { Intensity (flops/byte) }\end{array}$ \\
\hline Quadro_RTX & Intel Xeon E5-2630 v4 @ 2.20GHz & Quadro RTX 6000 & Turing & 16.3 & 624 & 26.12 \\
\hline Tesla_V100 (AWS P3) & Intel Xeon E5-2686 v4 @ 2.30GHz & Tesla V100-SXM2-16GB & Volta & 15.7 & 900 & 17.44 \\
\hline Tesla_P100 & Intel Xeon E5-2682 v4 @ 2.50GHz & Tesla P100-PCIE-16GB & Pascal & 9.3 & 732 & 12.70 \\
\hline Tesla P4 & Intel Xeon E5-2682 v4@ @2.50GHz & Tesla P4 & Pascal & 5.5 & 192 & 28.34 \\
\hline Tesla_M60 (AWS G3) & Intel Xeon E5-2686 v4 @ 2.30GHz & Tesla M60 & Maxwell & 4.8 & 160 & 30.12 \\
\hline
\end{tabular}

TABLE VIII

We use 55 TensorFlow models from MLPerf, AI-Matrix, and TensorFlow Slim, Detection Zoo, Deeplab for evaluation. These models ARE SORTED BY THE REPORTED ACCURACY AND SOLVE DIFFERENT TASKS: IMAGE ClASSIFICATION (IC), OBJECT DETECTION (OD), INSTANCE Segmentation (IS), Semantic Segmentation (SS), and Super Resolution (SR). We measured the PEaK throughput achieved on TESLA_V100 AND FIND THE OPTIMAL BATCH SIZE FOR EACH MODEL. ONLINE LATENCY IS DEFINED AS THE MODEL LATENCY FOR BATCH SIZE 1. GRAPH SIZE IS THE SIZE OF THE FROZEN GRAPH FOR A MODEL.

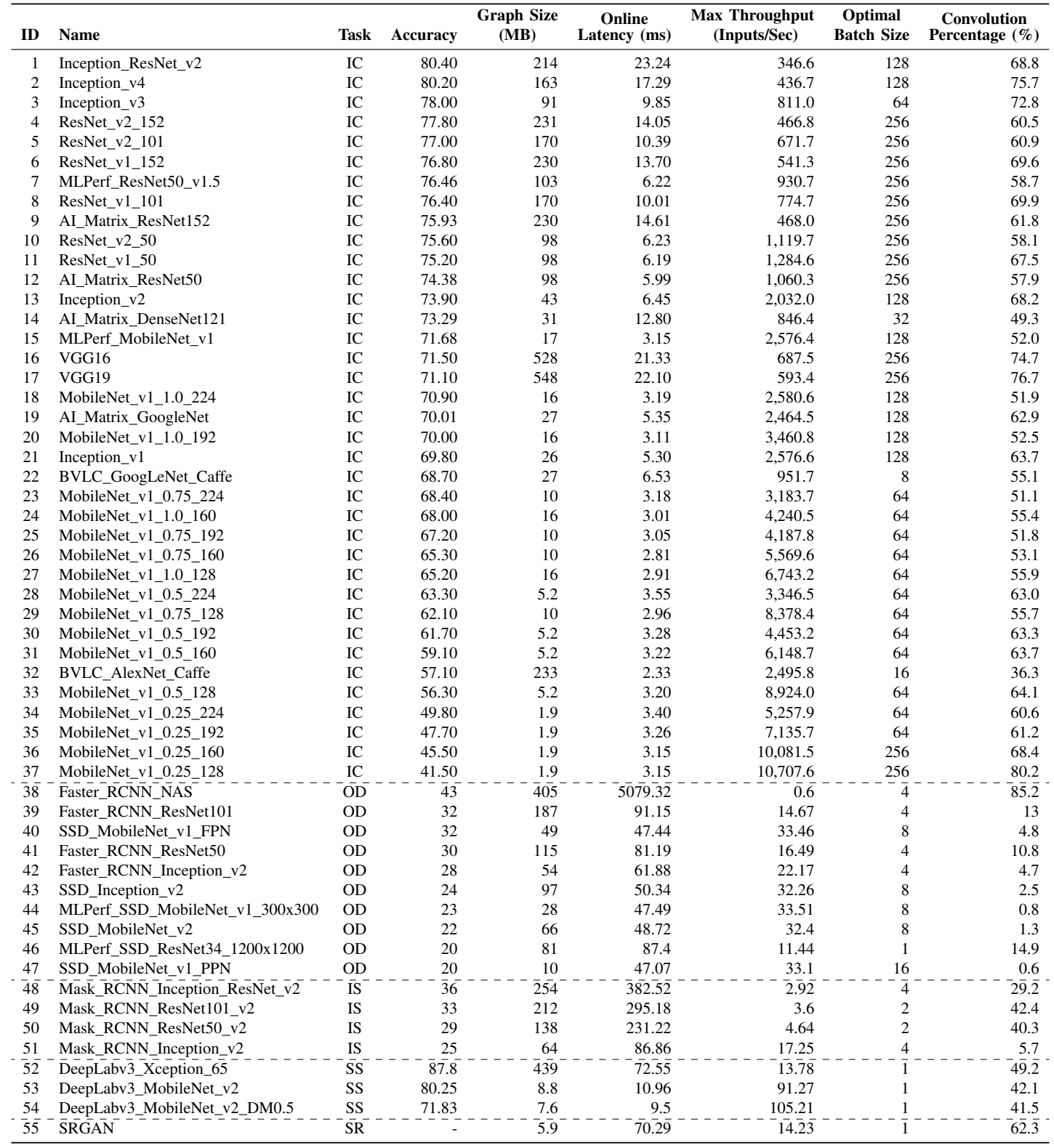


TABLE IX

IN-DEPTH CHARACTERIZATION OF THE 37 IMAGE CLASSIFICATION MODELS LISTED IN TABLE VIII AT THE OPTIMAL BATCH SIZES ON TES LA_V100. THE MODEL EXECUTION IS PARTITIONED INTO BEGINNING $(B)$, MIDDLE $(M)$, AND END $(E)$ INTERVALS BASED ON LAYER INDEX. THE MOST INTENSIVE STAGES FOR LATENCY, MEMORY ALLOCATION, FLOPS AND MEMORY ACCESS ARE SHOWN.

\begin{tabular}{|c|c|c|c|c|c|c|c|c|c|c|c|c|c|}
\hline ID & $\begin{array}{c}\text { Batch } \\
\text { Latency } \\
(\mathbf{m s})\end{array}$ & $\begin{array}{c}\text { GPU } \\
\text { Latency } \\
\text { Percentage } \\
(\%)\end{array}$ & $\begin{array}{c}\text { GPU } \\
\text { Gflops }\end{array}$ & $\begin{array}{c}\text { GPU } \\
\text { DRAM } \\
\text { Read } \\
\text { (GB) } \\
\end{array}$ & $\begin{array}{c}\text { GPU } \\
\text { DRAM } \\
\text { Write } \\
\text { (GB) }\end{array}$ & $\begin{array}{c}\text { GPU } \\
\text { Achieved } \\
\text { Occupancy } \\
(\%)\end{array}$ & $\begin{array}{c}\text { Arithmetic } \\
\text { Intensity } \\
\text { (Flops/byte) }\end{array}$ & $\begin{array}{c}\text { Arithmetic } \\
\text { Throughput } \\
\text { (TFlops) }\end{array}$ & $\begin{array}{l}\text { Memory } \\
\text { Bound? }\end{array}$ & $\begin{array}{c}\text { Latency } \\
\text { Stage }\end{array}$ & $\begin{array}{c}\text { Allocated } \\
\text { Memory } \\
\text { Stage }\end{array}$ & $\begin{array}{l}\text { flops } \\
\text { Stage }\end{array}$ & $\begin{array}{c}\text { Memory } \\
\text { Access } \\
\text { Stage }\end{array}$ \\
\hline 1 & 400.06 & 94.77 & $2,910.44$ & 50.64 & 38.74 & 39.74 & 32.56 & 7.68 & $x$ & M & M & M & $\mathrm{M}$ \\
\hline 2 & 324.49 & 93.92 & $2,492.92$ & 27.25 & 24.48 & 33.79 & 48.19 & 8.18 & $x$ & M & M & M & M \\
\hline 3 & 86.39 & 88.05 & 552.22 & 10.54 & 8.18 & 34.6 & 29.50 & 7.26 & $x$ & M & M & M & B \\
\hline 4 & 593.97 & 96.32 & $3,954.06$ & 58.90 & 65.44 & 43.51 & 31.80 & 6.91 & $x$ & E & E & M & E \\
\hline 5 & 412.37 & 94.90 & $2,725.14$ & 39.08 & 44.62 & 42.88 & 32.56 & 6.96 & $x$ & E & E & M & E \\
\hline 6 & 517.11 & 95.90 & $3,947.38$ & 51.17 & 54.77 & 42.78 & 37.26 & 7.96 & $x$ & $\mathrm{E}$ & E & M & $\mathrm{E}$ \\
\hline 7 & 275.05 & 92.43 & $1,742.39$ & 24.40 & 32.61 & 43.15 & 30.62 & 6.85 & $x$ & B & E & M & $\mathrm{E}$ \\
\hline 8 & 360.90 & 94.29 & $2,720.62$ & 33.87 & 37.12 & 42.19 & 38.32 & 7.99 & $x$ & $\mathrm{E}$ & $\mathrm{E}$ & M & $\mathrm{E}$ \\
\hline 9 & 591.47 & 96.29 & $4,034.74$ & 63.70 & 72.16 & 43.9 & 29.70 & 7.08 & $x$ & B & M & B & M \\
\hline 10 & 245.07 & 91.74 & $1,480.10$ & 21.84 & 28.29 & 42.96 & 29.52 & 6.58 & $x$ & $\mathrm{E}$ & $\mathrm{E}$ & M & $\mathrm{E}$ \\
\hline 11 & 213.52 & 90.42 & $1,477.33$ & 18.79 & 22.76 & 42.29 & 35.56 & 7.65 & $x$ & $\mathrm{E}$ & $\mathrm{E}$ & M & $\mathrm{E}$ \\
\hline 12 & 257.80 & 91.89 & $1,561.76$ & 24.86 & 33.39 & 44.26 & 26.81 & 6.59 & $x$ & B & M & B & M \\
\hline 13 & 68.27 & 83.62 & 363.33 & 9.67 & 7.32 & 40.23 & 21.38 & 6.36 & $x$ & B & B & M & B \\
\hline 14 & 40.24 & 93.32 & 150.02 & 10.13 & 7.93 & 44.94 & 8.30 & 4.00 & $\checkmark$ & B & B & B & B \\
\hline 15 & 51.57 & 79.76 & 148.18 & 7.08 & 6.81 & 52.58 & 10.67 & 3.60 & $\checkmark$ & M & M & M & M \\
\hline 16 & 399.31 & 94.98 & $2,655.39$ & 24.38 & 33.23 & 26.14 & 46.10 & 7.00 & $x$ & B & B & M & $\mathrm{E}$ \\
\hline 17 & 464.47 & 95.61 & $3,207.02$ & 26.44 & 37.65 & 24.91 & 50.04 & 7.22 & $x$ & B & B & M & $\mathrm{E}$ \\
\hline 18 & 51.59 & 79.73 & 148.18 & 6.97 & 6.75 & 52.59 & 10.80 & 3.60 & $\checkmark$ & M & M & M & M \\
\hline 19 & 56.08 & 80.20 & 259.14 & 7.63 & 6.18 & 42.16 & 18.76 & 5.76 & $x$ & M & B & M & B \\
\hline 20 & 38.48 & 79.55 & 108.93 & 6.51 & 6.19 & 52.32 & 8.58 & 3.56 & $\checkmark$ & M & M & M & B \\
\hline 21 & 53.35 & 79.43 & 252.06 & 7.21 & 5.61 & 41.74 & 19.67 & 5.95 & $x$ & M & B & M & B \\
\hline 22 & 9.08 & 80.00 & 20.26 & 0.73 & 0.84 & 33.87 & 12.97 & 2.79 & $\checkmark$ & $\mathrm{E}$ & B & $\mathrm{E}$ & B \\
\hline 23 & 20.82 & 73.14 & 45.10 & 4.86 & 4.11 & 52.73 & 5.03 & 2.96 & $\checkmark$ & M & M & M & M \\
\hline 24 & 14.92 & 78.26 & 38.17 & 3.24 & 2.88 & 48.92 & 6.23 & 3.27 & $\checkmark$ & M & M & M & M \\
\hline 25 & 15.69 & 72.61 & 33.10 & 3.52 & 3.08 & 52.02 & 5.01 & 2.91 & $\checkmark$ & M & M & M & M \\
\hline 26 & 11.30 & 71.86 & 23.14 & 2.31 & 2.17 & 51.01 & 5.17 & 2.85 & $\checkmark$ & M & M & M & M \\
\hline 27 & 9.86 & 77.23 & 24.39 & 1.90 & 1.84 & 47.78 & 6.54 & 3.20 & $\checkmark$ & M & M & M & M \\
\hline 28 & 20.00 & 71.93 & 52.03 & 2.99 & 2.85 & 43.87 & 8.91 & 3.62 & $\checkmark$ & B & M & B & M \\
\hline 29 & 7.75 & 71.35 & 14.80 & 1.26 & 1.35 & 47.12 & 5.68 & 2.68 & $\checkmark$ & M & M & M & M \\
\hline 30 & 15.07 & 71.75 & 38.22 & 2.08 & 2.09 & 43.27 & 9.17 & 3.53 & $\checkmark$ & B & M & B & M \\
\hline 31 & 10.91 & 71.38 & 26.62 & 1.29 & 1.42 & 41.43 & 9.83 & 3.42 & $\checkmark$ & B & M & B & M \\
\hline 32 & 6.52 & 68.69 & 15.36 & 0.76 & 0.51 & 37.31 & 12.11 & 3.43 & $\checkmark$ & B & B & B & B \\
\hline 33 & 7.44 & 70.48 & 17.05 & 0.71 & 0.88 & 39.88 & 10.73 & 3.25 & $\checkmark$ & B & M & B & M \\
\hline 34 & 11.95 & 53.93 & 14.79 & 1.25 & 1.42 & 44.25 & 5.52 & 2.30 & $\checkmark$ & B & M & B & M \\
\hline 35 & $\begin{array}{r}9.09 \\
9.06\end{array}$ & 53.68 & 10.87 & 0.84 & 1.02 & 43.46 & 5.82 & 2.23 & $\checkmark$ & B & M & B & M \\
\hline 36 & 25.36 & 60.78 & 36.75 & 3.26 & 3.09 & 42.39 & 5.79 & 2.38 & $\checkmark$ & B & M & B & M \\
\hline 37 & 23.71 & 70.01 & 23.81 & 1.87 & 2.31 & 39.8 & 5.69 & 1.43 & $\checkmark$ & M & M & B & M \\
\hline
\end{tabular}

TABLE X

Characterization of 10 MXNet Models, Which are Comparable to the TensorFlow ones Listed in Table ViII (LABELED With the SAME ID). THE ONLINE LATENCY IS MEASURED AT BATCH SIZE 1 AND THE OTHERS ARE MEASURED AT THE MODEL'S OPTIMAL BATCH SIZE ON TES LA_V100. THE ONLINE LATENCY AND MAXIMUM THROUGHPUT ARE NORMALIZED TO TENSORFLOW'S.

\begin{tabular}{|c|c|c|c|c|c|c|c|c|c|c|c|c|}
\hline ID & Name & $\begin{array}{c}\text { Normalized } \\
\text { Online } \\
\text { Latency }\end{array}$ & $\begin{array}{c}\text { Optimal } \\
\text { Batch } \\
\text { Size }\end{array}$ & $\begin{array}{c}\text { Normalized } \\
\text { Maximum } \\
\text { Throughput }\end{array}$ & $\begin{array}{c}\text { GPU } \\
\text { Latency } \\
\text { Percentage }\end{array}$ & $\begin{array}{c}\text { GPU } \\
\text { Gflops }\end{array}$ & $\begin{array}{c}\text { GPU } \\
\text { DRAM } \\
\text { Read } \\
\text { (GB) } \\
\end{array}$ & $\begin{array}{c}\text { GPU } \\
\text { DRAM } \\
\text { Write } \\
\text { (GB) } \\
\end{array}$ & $\begin{array}{c}\text { GPU } \\
\text { Achieved } \\
\text { Occupancy } \\
(\%)\end{array}$ & $\begin{array}{l}\text { Arithmetic } \\
\text { Intensity } \\
\text { (Flops/byte) }\end{array}$ & $\begin{array}{c}\text { Arithmetic } \\
\text { Throughput } \\
\text { (TFlops) }\end{array}$ & $\begin{array}{l}\text { Memory } \\
\text { Bound? }\end{array}$ \\
\hline 4 & ResNet_v2_152 & 1.76 & 256 & 1.03 & 97.00 & $4,116.42$ & 49.05 & 52.62 & 46.91 & 38.61 & 7.95 & $x$ \\
\hline 5 & ResNet_v2_101 & 1.59 & 256 & 1.02 & 96.77 & $2,882.65$ & 32.33 & 36.16 & 46.38 & 40.14 & 7.96 & $x$ \\
\hline 6 & ResNet_v1_152 & 1.68 & 256 & 0.90 & 96.20 & $3,828.11$ & 51.29 & 55.00 & 49.40 & 34.35 & 7.54 & $x$ \\
\hline 8 & ResNet_v1_101 & 1.60 & 256 & 0.91 & 95.67 & $2,589.76$ & 33.93 & 37.84 & 49.57 & 34.42 & 7.45 & $x$ \\
\hline 10 & ResNet_v2_50 & 1.41 & 256 & 1.03 & 97.10 & $1,636.10$ & 17.03 & 22.60 & 46.98 & 39.37 & 7.60 & $x$ \\
\hline 11 & ResNet_v1_50 & 1.32 & 256 & 0.96 & 94.90 & $1,339.50$ & 18.37 & 24.04 & 51.97 & 30.12 & 6.76 & $x$ \\
\hline 18 & MobileNet_v1_1.0_224 & 1.00 & 256 & 1.54 & 93.75 & 298.38 & 6.91 & 8.29 & 63.53 & 18.71 & 4.96 & $x$ \\
\hline 23 & MobileNet_v1_0.75_224 & 0.95 & 64 & 1.76 & 79.49 & 45.00 & 3.47 & 2.73 & 63.38 & 6.92 & 4.08 & $\checkmark$ \\
\hline 28 & MobileNet_v1_0.5_224 & 0.87 & 64 & 1.35 & 81.01 & 51.47 & 1.99 & 1.82 & 48.68 & 12.88 & 4.49 & $\checkmark$ \\
\hline 34 & MobileNet_v1_0.25_224 & 0.93 & 64 & 1.64 & 64.32 & 13.77 & 0.81 & 0.90 & 50.57 & 7.64 & 2.88 & $\checkmark$ \\
\hline
\end{tabular}

ers (Tensorflow's Conv2D and DepthwiseConv2dNative layers) with each model's optimal batch size on Tesla_V100. This is shown in the last column of Table VIII. We observe that: (1) the convolution layer latency percentage ranges between $36.3 \%$ and $80.2 \%$ for image classification models. This suggests that convolution layers still dominate (but not exclusively) the latency of image classification models - even on recent GPUs. This is not true for (2)object detection models, which (except for Faster_RCNN_NAS) attribute only $0.6 \%$ to $14.9 \%$ of latency to convolution layers. For these models, the dominating layer type is Where, which reshapes a tensor with respect to a user-defined operator. For (3)instance segmentation models, convolution layers dominate the model latency; except for Mask_RCNN_Inception_v2 whose latency is also dominated by where layers. For (4) semantic segmentation models, the model latency is affected by both the convolution layers and the memory-bound layers (such as Transpose, Add, and Mul). Finally, (5) the super resolution model SRGAN is dominated by convolution layers.

GPU latency, flops and memory accesses - Using the model-, layer-, and GPU kernel-level profiling, we perform an in-depth analyses of the 37 image classification models at 

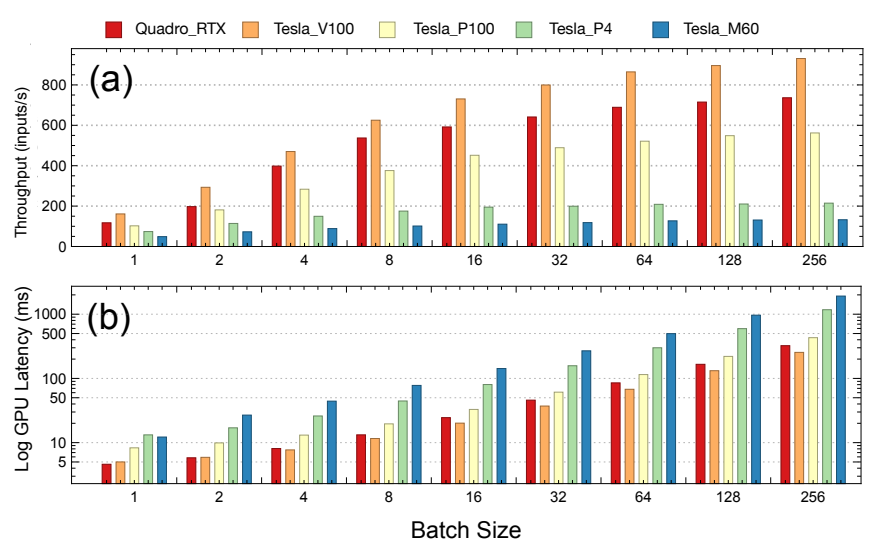

Fig. 11. The throughput and latency (log scale) of MLPerf_ResNet50_v1. 5 across batch sizes and systems.

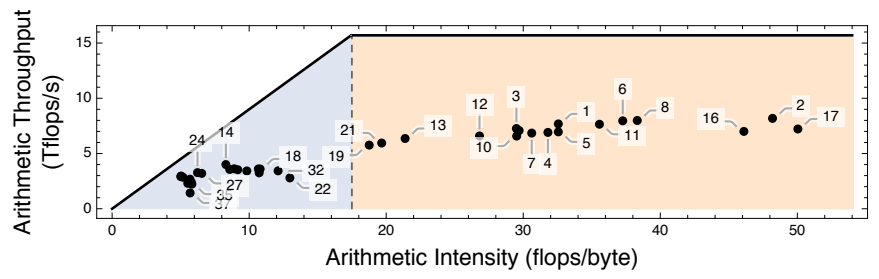

Fig. 12. The roofline analysis for the 37 image classification models with their the optimal batch sizes on Tesla_V100.

their optimal batch sizes on Tesla_V100. Table IX shows the models latency at the optimal batch size, GPU latency percentage (i.e. the latency due to GPU kernel execution normalized to the model latency), GPU metrics, and arithmetic intensity and throughput. It also shows the most intensive stage for latency, memory allocation, GPU flops, and memory access throughout the model execution. We find that across the models the GPU latency percentage varies from $53.68 \%$ to $95.61 \%$ and is roughly proportional to the number of flops and memory accesses (the sum of GPU DRAM reads and writes). We also observe that models with high batch latency tend to have a high GPU latency percentage. This either suggests that the GPU saturates for these models or that the models are not well optimized for GPU execution. The low GPU latency percentage for some models shows that the time spent within non-GPU code (framework overhead, GPU stalls due to synchronization, etc.) is high.

Batch size vs GPU achieved occupancy - The GPU achieved occupancy is a partial indicator of GPU utilization. Table VI shows that as a model's batch size approaches the optimal, its overall achieved GPU occupancy increases.

Roofline analysis - Figure 12 shows the roofline analysis for all 37 image classification models with their optimal batch sizes on Tesla_V100. Out of 37 models, 20 are memorybound. Models with low compute and memory requirements tend to be memory-bound and have lower accuracy, e.g. some variants of MobileNet which target edge devices. All models achieve at most $52 \%$ of the theoretical peak throughput, suggesting that there is room for optimizations.

Latency, memory allocation, flops, and memory access trend - To understand the performance trend within model execution, we divide the model execution into 3 intervals based on the layer index: beginning, middle, and end based on the layer index. We then compute the total latency, flops, and memory accesses within each interval and identify which interval dominates. The last 4 columns in Table IX show the results of the 37 image classification models on Tesla_V100. The demanding intervals vary across models and suggest that one can potentially interleave multiple model executions to increase GPU utilization.

\section{B. ML Framework Evaluation}

To compare ML frameworks, 10 MXNet models are selected from the MXNet model zoo [27]. We choose 6 variants of ResNet which are compute-intensive and are compute-bound (at the optimal batch size), and 4 variants MobileNet which are less compute-intensive and are memory-bound. The models (shown in Table $\mathrm{X}$ ) are comparable to the TensorFlow models. We perform the comparison between the TensorFlow and MXNet frameworks on Tesla_V100. The online latency and maximum throughput in the Table $\mathrm{X}$ are normalized to the corresponding values using TensorFlow. We use XSP to compute the optimal batch size for each MXNet model. Except for model 18, the optimal batch size for all MXNet models match the corresponding TensorFlow models.

Compute-bound models - Table $\mathrm{X}$ shows that the online latency (batch size 1) of MXNet ResNets is higher than that of the corresponding TensorFlow model. After looking into the analysis results, we find that while the total GPU kernel latencies of TensorFlow and MXNet ResNets are about the same, the MXNet ResNets have a much higher non-GPU latency. MXNet ResNet_v1_50, for example, has a nonGPU latency of $4.44 \mathrm{~ms}$ ( $55.1 \%$ of the total online latency) whereas it is only $2.18 \mathrm{~ms}$ for TensorFlow ResNet_v1_50 (35.3\% of the total). We observe that as the batch size increases (and the model becomes more compute-bound) the percentage of the non-GPU latency decreases and MXNet ResNets achieve about the same maximum throughput as TensorFlow ResNets. At the optimal batch size, TensorFlow and MXNet ResNets have comparable GPU latency percentage, flops, memory accesses, achieved occupancy, and roofline results. This suggests that MXNet incurs a fixed overhead for model execution which is more pronounced for small batch sizes.

Memory-bound models - For the less compute-intensive MobileNets, we observe that MXNet the MobileNets achieve the same online latency as the corresponding TensorFlow model. However, as the batch size increases (and the models become memory-bound) we find that MXNet MobileNets has fewer memory accesses and therefore a higher achieved GPU occupancy compared to the TensorFlow models. As a result, MXNetMobileNets achieve between $35 \%$ and $74 \%$ more throughput at their optimal batch sizes (shown in Table X). Further GPU kernel-level analysis attributes the cause to the Eigen library. The Eigen library is used by TensorFlow (but not MXNet) for element-wise layers and it incurs excessive DRAM reads and writes. This becomes a performance-limiting factor for memory-bound models. 


\section{System Evaluation}

We use XSP to evaluate MLPerf_ResNet50_v1.5 on all 5 GPU systems in Table VII using the NGC TensorFlow container. We fix the software stack (TensorFlow, cuDNN, cuBLAS, CUDA version, etc.) on all 5 systems to be the same. Figure 11a shows the throughput across systems and batch sizes. Figure $11 \mathrm{~b}$ shows the GPU latency (the total latency of all the GPU kernel calls) in log scale for the 5 systems across batch sizes. Although the Quadro_RTX GPU has a slightly higher peak FLOPS compared to Tesla_V100, it has a much lower memory bandwidth. Hence, Quadro_RTX straggles on memory-bound layers and performs slightly worse when compared to Tesla_V100. We observe that the performance at each batch size differs across systems. The performance also scales differently across systems with respect to the batch size.

Looking at the GPU kernel-level profile for each system, we find that the GPU kernels invoked are system-dependent — even with the same batch size and software stack. Both Quadro_RTX and Tesla_V100 call the same set of GPU kernels, while the other 3 systems use a different set of GPU kernels. This is because the same cuDNN API may use different GPU kernels for different GPU systems. For example, the convolution layers for batch size 256 on Tesla_P100, Tesla_P4, and Tesla_M60 invoke the maxwell_scudnn_* kernels, whereas on Quadro_RTX and Tesla_V100 the volta_scudnn_* kernels are invoked. This implies that cuDNN uses optimized kernels for GPU generations after Volta. Furthermore, because of the cuDNN algorithm selection heuristics, the distribution of the kernel calls differs across systems. For example, Tesla_V100 calls the volta_scudnn_128x64_relu_interior_nn_v1 kernel 34 times whereas Quadro_RTX calls it 18 times (the other 16 being dispatched to the volta_scudnn_128x128_relu_interior_nn_v1 kernel).

\section{CONCLuSion}

A big hurdle in optimizing and deploying ML workloads is understanding their performance characteristics across the HW/SW stack. The analyses currently performed on ML models and systems are largely limited by the lack of correlation between profiles from different profiling tools or methods. This paper proposes XSP, an across-stack profiling design that aggregates profile data from different sources and correlates them to construct a holistic and hierarchical view of ML model execution. While the across-stack profiling design is general, this paper focuses on how it enables in-depth automated profiling and characterization of ML models on GPUs. We use XSP's profiling and analysis capabilities to systematically characterize 65 state-of-the-art ML models. Through the 15 types of analysis introduced, we derive meaningful insights that would otherwise be difficult to discern without XSP. We show that XSP helps researchers understand the sources of inefficiency in ML models, frameworks, and systems.

\section{ACKNOWLEDGMENTS}

This work is supported by the IBM-ILLINOIS Center for Cognitive Computing Systems Research (C3SR) - a member of the IBM Cognitive Horizon Network, and the Applications Driving Architectures (ADA) Research Center - one of the JUMP Centers co-sponsored by SRC and DARPA.

\section{REFERENCES}

[1] “TensorFlow Profiler," www.tensorflow.org/api_docs/python/tf/profiler, accessed: 2020-02-20.

[2] "MXNet Profiler," mxnet.incubator.apache.org/api/python/profiler/profiler. html, accessed: 2020-02-20.

[3] "NVIDIA nvprof," docs.nvidia.com/cuda/profiler-users-guide/index.html, accessed: 2020-02-20.

[4] "NVIDIA Nsight," developer.nvidia.com/tools-overview, accessed: 2020$02-20$.

[5] "NVIDIA GPU-Accelerated Containers," www.nvidia.com/en-us/ gpu-cloud/containers/, accessed: 2020-02-20.

[6] "NVIDIA Tools Extension," docs.nvidia.com/cuda/profiler-users-guide/ index.html\#nvtx, accessed: 2020-02-20.

[7] "MLPerf Inference," github.com/mlperf/inference, accessed: 2020-02-20.

[8] W. Zhang, W. Wei, L. Xu, L. Jin, and C. Li, "AI Matrix: A Deep Learning Benchmark for Alibaba Data Centers," 2019.

[9] "NVIDIA CUPTI," developer.nvidia.com/cuda-profiling-tools-interface, accessed: 2020-02-20.

[10] "NVTX Plugins for Deep Learning," github.com/NVIDIA/nvtx-plugins, accessed: 2020-02-20.

[11] S. Chintala, "ConvNet Benchmarks," github.com/soumith/ convnet-benchmarks, accessed: 2020-02-20.

[12] Baidu, "Deepbench," github.com/baidu-research/DeepBench, accessed: 2020-02-20.

[13] C. Li, A. Dakkak, J. Xiong, and W.-M. Hwu, "Benanza: Automatic $\mu$ Benchmark Generation to Compute "Lower-bound" Latency and Inform Optimizations of Deep Learning Models on GPUs." IEEE, May 2020, the 34th IEEE International Parallel \& Distributed Processing Symposium (IPDPS'20).

[14] T. Ben-Nun, M. Besta, S. Huber, A. N. Ziogas, D. Peter, and T. Hoefler, "A Modular Benchmarking Infrastructure for High-Performance and Reproducible Deep Learning." IEEE, May 2019, the 33rd IEEE International Parallel \& Distributed Processing Symposium (IPDPS'19).

[15] "Trace Context," www.w3.org/TR/trace-context, accessed: 2020-02-20.

[16] “Open Tracing," opentracing.io, 2020, accessed: 2020-02-20.

[17] "Open Telemetry," opentelemetry.io, accessed: 2020-02-20.

[18] A. Pal and M. Pal, "Interval tree and its applications," Advanced Modeling and Optimization, vol. 11, no. 3, pp. 211-224, 2009.

[19] "Amazon EC2 P3 Instances," aws.amazon.com/ec2/instance-types/p3/, accessed: 2020-02-20.

[20] "NVIDIA GPU Metrics Reference," docs.nvidia.com/cuda/ profiler-users-guide/index.html\#metrics-reference, accessed: 202002-20.

[21] S. Williams, A. Waterman, and D. Patterson, "Roofline: An insightful visual performance model for floating-point programs and multicore architectures," Lawrence Berkeley National Lab.(LBNL), Berkeley, CA (United States), Tech. Rep., 2009.

[22] "NVIDIA cuDNN," developer.nvidia.com/cudnn, accessed: 2020-02-20

[23] G. Guennebaud, B. Jacob et al., "Eigen v3," eigen.tuxfamily.org, accessed: 2020-02-20

[24] "TensorFlow-Slim Image Classification Model Library," github.com/ tensorflow/models/tree/master/research/slim, accessed: 2020-02-20.

[25] "TensorFlow Detection Model Zoo," github.com/tensorflow/models/ blob/master/research/object_detection/g3doc/detection_model_zoo.md, accessed: 2020-02-20.

[26] "TensorFlow DeepLab Model Zoo," github.com/tensorflow/models/blob/ master/research/deeplab/g3doc/model_zoo.md, accessed: 2020-02-20.

[27] "MXNet Gluon Model Zoo," gluon-cv.mxnet.io/model_zoo/index.html, 2020, accessed: 2020-02-20. 